\title{
A Giant Left Atrial Diverticulum Diagnosed by Transesophageal Echocardiogram and Multidetector Computed Tomography: A Case Report and Brief Review of the Literature
}

\author{
Qiang Chen, MD,${ }^{1}$ Hua Cao, MD,${ }^{1}$ Gui-Can Zhang, MD, ${ }^{1}$ Xin-Ming Huang, $\mathrm{MD}^{2}$ \\ Departments of ${ }^{1}$ Cardiovascular Surgery and ${ }^{2}$ Radiology, Union Hospital, Fujian Medical University, Fuzhou, China
}

\section{ABSTRACT}

Giant left atrial diverticulum is a rare congenital malformation that is most commonly diagnosed in incidental finding. Here, we reported a case of a 1-year-old asymptomatic child with a giant left atrial diverticulum. Diagnosis was established by transthoracic echocardiography (TTE), and confirmed by multidetector computed tomography (MDCT) and transesophageal echocardiography (TEE). We excised the diverticulum and sewed its base under cardiopulmonary bypass and cardiac arrest. The patient had a good postoperative result.

\section{INTRODUCTION}

Diverticula of the left atrium are clinical entities rarely seen among congenital cardiac abnormalities, and their etiology is unknown. We report a case of a giant left atrial diverticulum of a 1-year-old asymptomatic child, who was diagnosed through an incidental physical examination. After sufficient preoperative preparation, the patient underwent resection of the diverticulum, with a satisfactory clinical result.

\section{CASE REPORT}

A 1-year-old asymptomatic boy was admitted to our department after a local hospital visit. Transthoracic echocardiogram showed a pericardial cyst during a respiratory tract infection. The patient had been growing well, received routine physical examinations and blood workup, and biochemical tests showed each index was normal. Auscultation revealed no murmur on the chest. Transthoracic echocardiography identified an accessory giant left atrial diverticulum, which was bordered by a thin wall in continuity with the left atrium that lead to the dextroposition of the heart. The diagnosis was confirmed by

Received August 25, 2016; accepted February 5, 2017.

This research was sponsored by Chinese National and Fujian provincial key clinical specialty construction programs.

Correspondence: Qiang Chen, MD, Department of Cardiovascular Surgery, Union Hospital, Fujian Medical University, Xinquan Road \#29, Fuzhou, 350001, China; 0086-13348286041; fax: 0086-591-83344034 (e-mail: chenqiang2228@163.com). multidetector computed tomography, and the size of the diverticulum was about $50 \times 50 \mathrm{~mm}$ (Figure 1). From the CT image, the diverticulum communicated with the left atrium but not the left ventricle, and extended toward the left and right ventricle, compressing them. Owing to this compression and its risk of progression, combined with the risk of thromboembolism, surgical excision was considered.

After general anesthesia, transesophageal echocardiography confirmed the diagnosis of the giant left atrial diverticulum and was used to exclude left atrial thrombus and mitral regurgitation (Figure 2). Through a median sternotomy, upon opening and suspending the pericardium, the big size of the left atria diverticulum was noted to extend over the left and the right ventricle (Figure 3). Under cardiopulmonary bypass and cardiac arrest, the diverticulum was opened (Figure 4). There were no thrombi in the cavity. Surgery involved resection of the part of the diverticulum and stitching its base of the left atrial wall (Figure 5). Pathological evaluation of the surgically excised portion of the diverticulum demonstrated mainly fibrous and elastic tissue, and adipose tissue (Figure 6).

\section{DISCUSSIO}

Diverticula of the left atrium are rare malformations of unknown etiology.

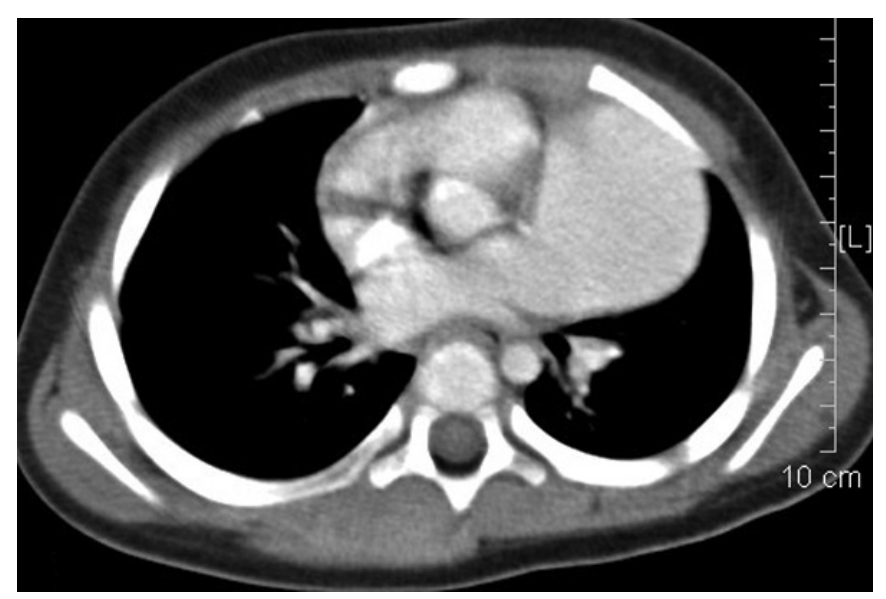

Figure 1. MDCT showed the diverticulum communicating with the left atrium. 


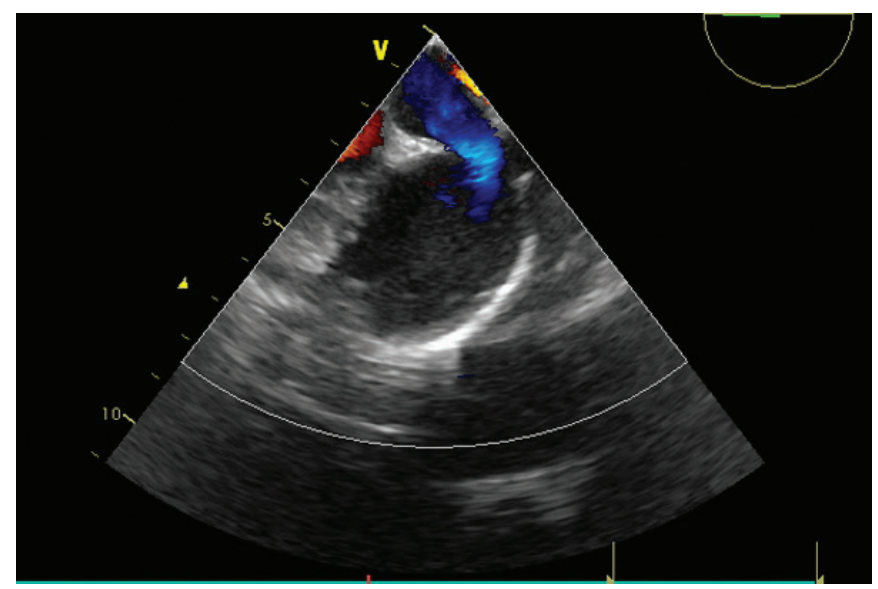

Figure 2. Intraoperative TEE showed the diverticulum.

Clinically, diverticula of the atrium usually present asymptomatically, but can also be associated with arrhythmias, thromboembolism, mitral valve regurgitation, or occasionally compression of surrounding structures [Kim 1995]. Pitts et al made the first report about left atrium diverticulum in March of 1962, which is the projecting portion like a cyst from the heart cavity to outside the pericardial cavity [Pitts 1962]. Left atrium diverticula may be single or multiple, located in the anterior wall, posterior wall or the superior wall of left atrium, like a cyst or tube. Incedayi and his colleagues characterized the frequency and location of left atrial diverticula by using multidetector computed tomography (MDCT). They concluded left atrial diverticula are frequently detected during routine cardiac computed tomography angiography examinations [Incedayi 2012].

Giant left atrial diverticulum has been described sporadically in several studies and case reports, in most of which an isolated left atrial diverticulum resection was performed. Lei et al reported the case of an 18-year-old woman who presented

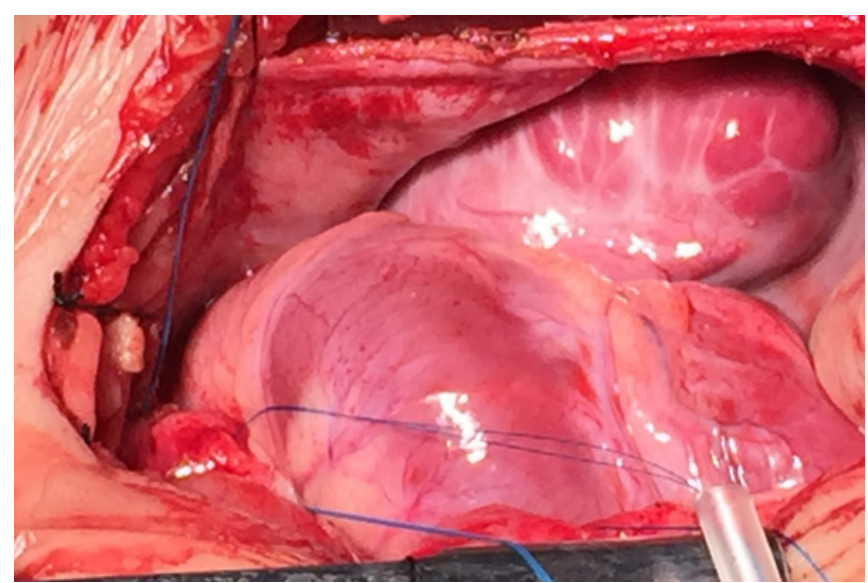

Figure 3. The diverticulum was exposed in the operation, which compressed the left ventricle.

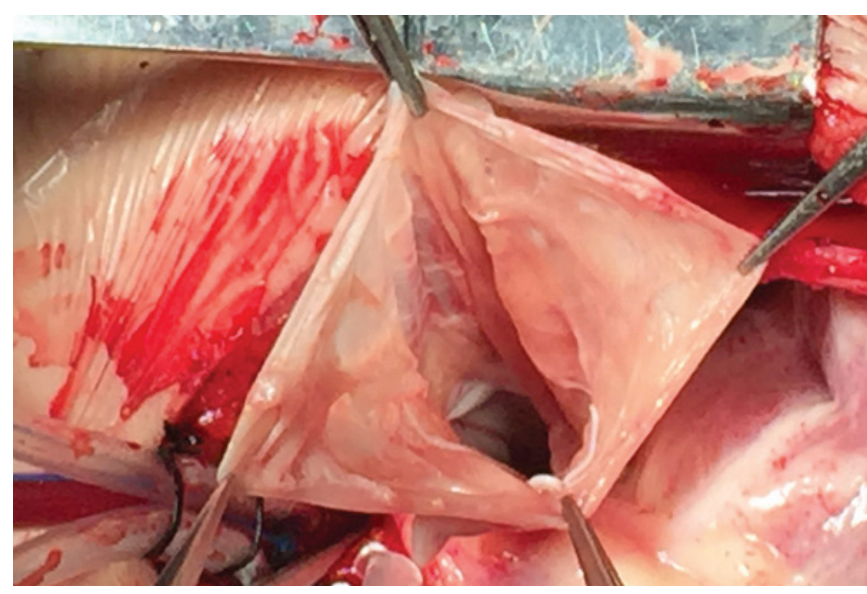

Figure 4. The diverticulum was opened with cardiac arrest.

with a $12 \times 7 \mathrm{~cm}$ left atrial diverticulum, and the patient had an uneventful recovery after a surgical resection [Lei 2014]. Gao et al reported a 23-year-old female with a giant left atrium diverticulum $(11 \times 10 \mathrm{~cm})$. The diverticulum was excised by oversewing its base using a side-biting clamp off bypass [Gao 2011]. Nomura and his colleagues reported a 4-year-old girl with a giant left atrial diverticulum $(7.2 \times 3.5 \mathrm{~cm})$ and severe mitral regurgitation, in which the patient underwent resection of the diverticulum, plication of the left atrium wall, and mitral valve replacement [Nomura 2008]. McGuinness et al reported the case of an asymptomatic 11/2-year-old girl who received three surgeries. In the first, through a left posterolateral thoracotomy, only an elective atrioplasty was undertaken. During the second, the diverticulum was excluded from the circulation through pericardial patch closure of its orifice from the left atrium with cardiopulmonary bypass. But a left dominant coronary artery containing a critical dynamic stenosis of the circumflex artery occurred. Following, extracorporeal membrane oxygenation was needed for life support. In the third, further surgical repair was undertaken involving pledget supported sutures from the atrioventricular groove

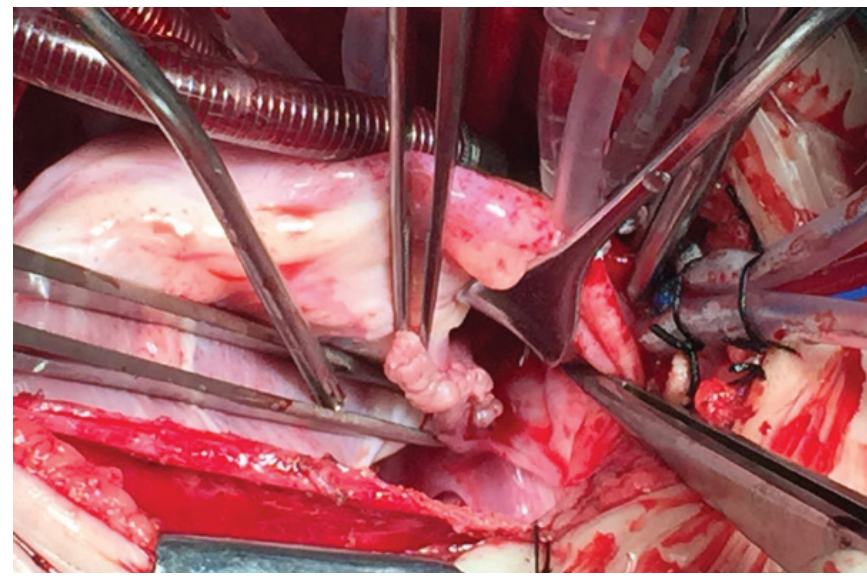

Figure 5. The diverticulum was excised. 


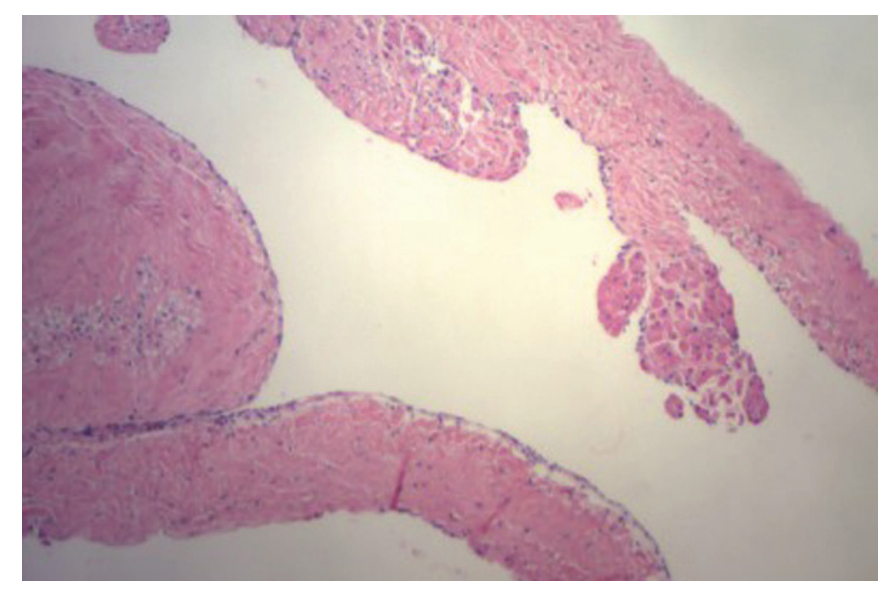

Figure 6. Histology demonstrated mainly fibrous and elastic tissue, and adipose tissue.

to the apex of the left ventricle [McGuinness 2007]. The united examination of MDCT and echocardiography made the diagnosis of left atrial diverticulum easier. The left atrial diverticulum must be distinguished from pericardial defect, pericardiac cyst, dilated cardiomyopathy, left atrial dilatation caused by mitral stenosis, and so on [Wan 2009]. Our case was misdiagnosed as a pericardial cyst in the local hospital, then correctly diagnosis in our hospital by the MDCT and echocardiography examination. For larger left atrial diverticula, no matter whether there are any symptoms, early surgical excision is the therapy of choice to renew the atrium normal shape, remove arrhythmias, decrease systemic thrombosis, and improve cardiac function, if necessary. So, surgical diverticulum excision was the course chosen in our case to good effect.

\section{REFERENCES}

Gao C, Wang R, Wang G, Wang Y. 2011. Giant left atrial diverticulum. J Card Surg 26:70.

Incedayi M, Öztürk E, Sonmez G, et al. 2012. The incidence of left atrial diverticula in coronary CT angiography. Diagn Interv Radiol 18:542-6.

Kim YJ, Kim H, Choi JY. 1995. Right atrial aneurysm. Cardiol Young 5:354-6.

Lei Q, Guo HM, Luo ZC. 2014. Surgical treatment of giant left atrial diverticulum in an adult. Ann Thorac Surg 98:1820-1.

McGuinness J, Kindawi A, Tajri S, Walsh K, Nolke L, Wood AE. 2007. Surgical management of giant left atrial diverticulum. J Thorac Cardiovasc Surg 133:820-2.

Nomura K, Matsumura Y, Shinohara G, Nakamura Y. 2008. A 4-year-old girl with giant left atrial diverticulum resulting in severe mitral regurgitation. Cardiovasc Pathol 17:254-5.

Pitts RM, Potts W. 1962. Congenital diverticulum of the left atrium. Arch Surg 84:334-6.

Wan Y, He Z, Zhang L, et al. 2009. The anatomical study of left atrium diverticulum by multi-detector row CT. Surg Radiol Anat 31:191-8. 